\title{
Os desafios para manejo da pandemia da Covid-19 em um hospital de Belo Horizonte e sua relação com a APS
}

\author{
Alzira Oliveira Jorge, Monica Aparecida Costa, Viviane Reis Couto
}

\section{RESUMO}

0 artigo objetiva uma análise crítica dos desafios para o enfrentamento da pandemia do Coronavírus Sars-Cov2 a partir da vivência em hospital de caráter regional da parte norte da Região Metropolitana de Belo Horizonte. Além da caracterização da organização hospitalar e das articulações existentes com as estações cuidadoras do SUS-BH, é abordado o conjunto de ações adotadas para prevenção, controle e assistência aos pacientes e trabalhadores no contexto da Covid-19 e as lições aprendidas. Aprendizado esse relacionado à importância da agilidade institucional para o desenvolvimento das adequações estruturais e organizacionais para manejo da nova doença e à comunicação como ferramenta de transparência para envolvimento das equipes. Apesar da articulação com o SUS-BH das ações planejadas, identifica-se também a necessidade da ampliação de estratégias junto à Atenção Básica para a continuidade do cuidado aos pacientes egressos hospitalares com diagnóstico de Covid-19.

Palavras-chave: Infecções por Coronavirus, pandemias, Redes Comunitárias, Assistência à Saúde, Sistema Único de Saúde.

\section{ABSTRACT}

The article aims at a critical analysis of the challenges of the Coronavirus Sars-Cov2 pandemic from the experience of a regional hospital in the northern part of the Metropolitan Region of Belo Horizonte. In addition to the characterization of the hospital organization and the existing links with SUS-BH caregiving stations, it also addressed the set of actions adopted and lessons learned from the prevention, control and assistance of patients and workers in the context of Covid-19. This learning experience is related to the importance of institutional agility for the development of structural and organizational adaptations on the management of the new disease and communication as a transparency tool for team involvement. Besides the articulation with the planned actions with SUS-BH, it also identified the need to expand strategies with Primary Health Care for the continuity of care for patients discharged from hospitals diagnosed with Covid-19.

Keywords: Coronavirus Infections, Pandemics, Community networks, Delivery of Health Care, Unified Health System.

\section{Revista da Rede APS 2021}

Publicada em: 01/04/2021

DOI:10.14295/aps.v3i1.143

Alzira Oliveira Jorge

(Médica e Professora da

Faculdade de Medicina da UFMG e Diretora Geral do Hospital Risoleta Tolentino Neves - HRTN de Belo Horizonte, MG, Brasil.)

Monica Aparecida Costa (Médica sanitarista, mestre em ciências da saúde e Diretora Técnico-Assistencial do HRTN, Belo Horizonte, MG, Brasil)

Viviane Reis Couto (Enfermeira, Especialista em Gestão de Saúde e Assessora técnica do Hospital Risoleta Tolentino Neves, Belo Horizonte, MG, Brasil)

Correspondência para:

Alzira Oliveira Jorge alzira.jorge@hrtn.fundep.ufmg.br 


\section{INTRODUÇÃO}

Este artigo pretende analisar os desafios colocados para o enfrentamento da pandemia do Coronavírus Sars-Cov2 a partir da experiência vivenciada no Hospital Risoleta Tolentino Neves (HRTN), que possui atuação regionalizada na Rede de Atenção à Saúde da parte norte da Região Metropolitana de Belo Horizonte (RMBH). É feita uma caracterização dessa instituição, suas articulações com as demais estações cuidadoras do Sistema Único de Saúde de Belo Horizonte (SUS-BH), as ações adotadas para prevenção, controle e assistência de pacientes e de trabalhadores no contexto da pandemia e o aprendizado decorrente desse complexo processo.

Este Hospital pertencente à Secretaria de Estado da Saúde de Minas Gerais (SES-MG), foi descentralizado para a gestão de Belo Horizonte (BH) e é gerenciado pela Fundação de Desenvolvimento da Pesquisa (FUNDEP) e Universidade Federal de Minas Gerais (UFMG) desde junho de 2006. Atua como o principal hospital do eixo norte de $\mathrm{BH}$ e Região Metropolitana, sendo referência de Urgência e Emergência para 1,2 milhões de habitantes com grande vulnerabilidade econômica e social. Todas as atividades assistenciais são gratuitas e a clientela é $100 \%$ atendida por meio do SUS. 0 HRTN constitui-se em Instituição de Ensino, Pesquisa e Extensão com múltiplos cenários de práticas para alunos de cursos técnicos, graduação e pós-graduação, beneficiando cerca de 2000 estudantes e residentes em seus campos de estágio a cada ano.

O modelo institucional integra a assistência, a gestão, o ensino e a pesquisa e está fundamentado em quatro Linhas de Cuidado (LC): Clínica, Cirúrgica, Materno Infantil e Intensiva, definidas conforme as necessidades de saúde da população da área de abrangência (FRANCO; MAGALHÃES Jr, 2003; COSTA et al, 2010). Possui o Pronto Socorro e a Maternidade como portas de entrada para os atendimentos de Urgência e Emergência, com funcionamento ininterrupto e base territorial e geográfica definida para a assistência do trauma, das doenças clínico-cirúrgicas, neurológicas e vasculares de média e alta complexidade e para as gestantes de alto, médio e risco habitual, possuindo capacidade operacional de 383 leitos. Cerca de $60 \%$ dos pacientes atendidos no Pronto Socorro são originários de Belo Horizonte e $40 \%$ são de outros municípios. Nos atendimentos da Maternidade verifica-se um maior número de mulheres das cidades vizinhas, chegando a quase $60 \%$, por ser a única referência hospitalar. Os principais municípios que utilizam o HRTN são Ribeirão das Neves, Santa Luzia, Vespasiano, Lagoa Santa, Pedro Leopoldo e Confins. Essa base territorial mantém-se ao longo dos anos e está relacionada à oferta insuficiente, em algumas linhas de cuidado, nos sistemas de saúde dessas cidades.

A grande vulnerabilidade da população se expressa na complexidade dos perfis de adoecimento e morte da clientela atendida, desafiando os modelos tradicionais de abordagem do cuidado em saúde baseados na lógica das profissões e numa concepção de assistência hospitalocêntrica, desconectada da articulação em rede. É fundamental, portanto, o desenvolvimento de um processo de interação e integração do HRTN com as Unidades que compõem a Rede de Atenção à Saúde do eixo norte de BH e RM para assegurar a integralidade e a longitudinalidade da assistência (SILVA, 2014).

Essa racionalidade busca superar os modelos tradicionais de gestão e cuidado e se manifesta também na dinâmica interna com a transversalização da Política da Humanização, ampliação da democratização por meio de fóruns ampliados de planejamento e cogestão, além da produção de relações interprofissionais e interequipes mais horizontalizadas e solidárias (CECÍLIO; MERHY, 2010) e que incluem parcerias com as instâncias de controle sociais como o Conselho Local de Saúde, Conselhos Distritais e Conselhos Municipal de BH e Estadual de Saúde.

Em termos de produção assistencial, no primeiro semestre de 2020, o HRTN realizou média mensal de 5.700 consultas de urgência, 1.400 internações, 450 cirurgias, 250 partos com taxa de cesárea de $23,0 \%$ e 2.800 consultas 
ambulatoriais para acompanhamento de pacientes de perfil cirúrgico.

São múltiplas as parcerias entre o hospital e as unidades das Redes de Atenção à Saúde e de Assistência Social de BH e municípios vizinhos desenvolvidas para possibilitar maior resolutividade das ações desenvolvidas, considerando a complexidade das situações de grande vulnerabilidade da clientela atendida. 0 HRTN atua como referência assistencial, contribui para o processo de qualificação técnica das equipes de saúde regionais e desenvolve matriciamento para $\mathrm{BH}$ e cidades próximas.

A gestão criteriosa dos processos de desospitalização é fundamental para potencializar as articulações entre os dispositivos das redes de saúde e de assistência social, considerando que as discussões para uma alta oportuna e segura trazem à tona uma confluência de fatores. Nessa perspectiva, a articulação promovida pelas duas Equipes de Atenção Domiciliar (EMAD's) existentes no HRTN junto às equipes das LC, seja por busca ativa ou por interconsulta, gerou resultados expressivos no processo de vinculação dos usuários incluídos pelas EMAD's dos 9 Distritos Sanitários de $\mathrm{BH}$, dos municípios vizinhos ou das próprias do hospital.

Como exemplo do potencial de abordagem em rede, vale mencionar a experiência desenvolvida na LC Materno Infantil/Rede Cegonha, iniciada por aproximação dos níveis gestores da Maternidade e Direção do Hospital com o Distrito Sanitário Venda Nova (DISAVN) por meio de reuniões mensais conjuntas, resultando em diversas parcerias. Salienta-se a atuação da equipe do HRTN na condução do Pré-Natal de Alto Risco (PNAR) para as gestantes no Centro de Especialidades do DISAVN com consultas e exames, garantia de internações clínicocirúrgicas para as intercorrências, discussão prévia dos casos entre as equipes, realização de visitas prévias de reconhecimento do ambiente e modelo de parto humanizado para as gestantes vinculadas aos Centros de Saúde e ao PNAR, e referenciamento das puérperas e dos recém-nascidos para as Unidades Básicas de Saúde. Vale ressaltar a participação de médicos e enfermeiros do HRTN nos processos de capacitação das equipes dos centros de saúde do DISAVN no curso de Pré-Natal Baseado em Evidências, assim como a atuação da equipe da Regional no Projeto de Aprimoramento e Inovação no Cuidado e Ensino em Obstetrícia e Neonatologia - APICE ON - desenvolvido por iniciativa do Ministério da Saúde, no Hospital Risoleta, de 2018 a 2020.

Dentre os perfis de gestantes e puérperas atendidas, destacam-se as de grande vulnerabilidade como adolescentes, mulheres com trajetória nas ruas, usuárias de álcool e outras drogas ou em processo de desagregação familiar. Tais situações são abordadas de maneira integrada, a partir da construção conjunta do caso com os dispositivos da Rede de Saúde, Assistência Social, Conselhos Tutelares e movimentos sociais de defesa da mulher de $\mathrm{BH}$ e dos municípios vizinhos, buscando o empoderamento dessas mulheres, resgate da sua autonomia/dignidade e preservação do vínculo com o recém-nascido.

Outra iniciativa é o Ambulatório de Controle de Anticoagulação que funciona no HRTN como referência para pacientes dos Centros de Especialidades Médicas de Venda Nova e da Regional Norte, responsável pelo monitoramento clínico-laboratorial de vários perfis de pacientes por meio de equipe multiprofissional.

Experiências no processo de matriciamento também se afirmam na articulação com as unidades de saúde do eixo norte da RMBH como a capacitação no Protocolo de Abordagem Multiprofissional dos Pacientes com Pé Diabético desenvolvida junto aos profissionais de diversos municípios vizinhos e originários da atenção básica, UPA's, centros de especialidades, hospitais e gestores locais do SUS regional, propiciando a discussão e comanejo de pacientes complexos com definição de atribuições claras sobre as responsabilidades e resolutividade de cada nível assistencial para um cuidado integral e longitudinal.

Outra parceria inovadora foi a desospitalização negociada e de transferência regulada para 
hospitais de menor complexidade dos municípios vizinhos com pacientes internados no HRTN, com estabilidade clínica e que estavam aguardando ajustes nos medicamentos e transição de farmacoterapia injetável para oral.

Essa transferência do cuidado ocorreu após consenso de projeto terapêutico e garantia da contra referência em caso de necessidade de estabilização ou de agravamento clínico dos usuários.

Em cada Linha de Cuidado, o processo de desospitalização busca a negociação com a rede, antes da alta, de um plano de cuidados que considere as necessidades para estabilidade clínica dos doentes, evitando-se o processo de agravamento e reinternação. É fundamental que pacientes portadores de condições que demandem acompanhamento, não sejam liberados sem articulação institucional com as outras unidades. Tal desafio apresenta-se especialmente nas situações de sofrimento mental, de violência contra crianças, adolescentes, idosos e mulheres e nas condições de dependência química, quando a sintonia de trabalho e da concepção de assistência integral das equipes é fundamental para superar os paradigmas que abordam as condições de urgência de maneira restrita à queixa.

No ano de 2020 iniciou-se o desenvolvimento de projetos de aperfeiçoamento do modelo de governança clínica, políticas de segurança e qualidade assistenciais, incorporação tecnológica, recomposição do parque tecnológico de equipamentos e de informatização, visando a configuração do HRTN como hospital digital e paperless.

Entretanto, em março, a agenda foi invadida pela premência do enfrentamento da Pandemia do novo Coronavírus no país, alterando a ordem das prioridades organizacionais e exigindo o planejamento e a concretização de um plano que possibilitasse o manejo adequado da COVID-19 neste território.

\section{O DESAFIO DO ENFRENTAMENTO DA EPIDEMIA NO HRTN}

Como estratégia estruturante, destaca-se a construção do Plano de Contingência para Enfrentamento à Pandemia da COVID-19 no HRTN de maneira articulada às Políticas e Diretrizes estaduais e municipais. Várias medidas foram adotadas para assegurar as condições técnicas e organizacionais de proteção e segurança aos pacientes, acompanhantes e equipes. Em 16 de março de 2020 foi criado o Comitê de Enfrentamento à COVID 19 com a presença da diretoria, lideranças das LC e apoios estratégicos e sua conformação interna contemplou o desenvolvimento de três eixos: Reestruturação Assistencial, Segurança e Assistência aos Trabalhadores e Informação e Comunicação.

No eixo da atenção à saúde foi implantada a Linha de Cuidado aos Pacientes de COVID-19, com a organização de três áreas de atendimento separadas dos demais setores assistenciais com segregação de fluxo de pacientes sintomáticos respiratórios, adequação das instalações e equipamentos, atuação de equipe multiprofissional exclusiva e adoção das medidas de proteção ambiental e individual. $O$ Centro de Atendimento de SRAG/COVID (CECOVID) foi aberto em 30/03 como a porta de entrada 24 horas para atendimento de sintomáticos respiratórios adultos e gestantes sem evidência de urgência obstétrica, possuindo estrutura de leitos para manejo inicial de doentes de maior instabilidade e complexidade. As Enfermarias de Coorte para Pacientes Adultos e Gestantes com Suspeita de SRAG/COVID 19 foram organizadas em duas das alas anteriormente utilizadas para internação de pacientes cirúrgicos com 24 leitos, totalizando 48 leitos. No caso das gestantes com urgência obstétrica ou para puérperas suspeitas e seus recém-nascidos foi organizado um Alojamento Conjunto de Coorte COVID-19 na Maternidade. Por fim, o Centro de Tratamento Intensivo (CTI) de Coorte foi estruturado a partir do remanejamento de 11 leitos já existentes e depois ampliado com mais 8 leitos, totalizando 19 leitos. 
É importante ressaltar que essa reorganização assistencial foi feita de maneira articulada as Redes de $\mathrm{BH}$ e RM, preservando o papel do HRTN como hospital geral, preservando o cuidado às gestantes e pacientes clínicocirúrgicos não COVID. Nessa nova organização, fluxos e processos de trabalho foram revistos, protocolos foram elaborados e realizadas capacitações sem que nenhum serviço assistencial fosse fechado ou reduzido, evitando-se prejuízos aos usuários ou sobrecarga da Rede de Atenção à Saúde.

No eixo da Segurança e Assistência aos Trabalhadores, as medidas priorizaram estratégias para proteção e cuidado dos profissionais. Apesar das dificuldades conjunturais de sobrepreço e falta de itens, conseguiram-se estoques sustentáveis de Equipamentos de Proteção Individual (EPI), permitindo um modelo de uso baseado no risco de exposição e vinculado aos processos de capacitação e monitoramento em cada setor. Estabeleceram-se critérios para afastamento e/ou para home office, suspensão ou antecipação de férias e compensação de banco de horas, medidas para manejar os riscos préexistentes e evitar a menor circulação de trabalhadores no ambiente institucional. Em caso de suspeição de COVID-19, assegurou-se o fluxo para avaliação médica e realização de diagnóstico no CECOVID em parceria com o Serviço Especializado de Medicina e Segurança do Trabalho (SESMT). Outras ações foram mudanças no Refeitório com entregas de alimentação por setor, demarcação de sinalizações no chão e ambientes de convivência e definição de rotas específicas para movimentação de suspeitos de COVID-19 para as áreas de coorte.

Um grande investimento institucional ocorreu para o apoio emocional das equipes, expostas à grande pressão colocada pela Pandemia, criando-se possibilidades de acolhimento com abordagem terapêutica, seja psicológica por consultas individuais ou grupos operativos, seja por atendimento homeopático, oficinas com técnicas de mindfulness ou pelo acompanhamento habitual do SESMT/HRTN. A construção desta rede de apoio aos funcionários permitiu maior conforto para enfrentamento das tensões e maior segurança nos ambientes de trabalho.

Buscando melhor comunicação interna e transparência foram produzidos materiais informativos para esclarecer, capacitar e divulgar ações desenvolvidas para os atores institucionais e comunidade, especialmente os usuários do Conselho Local de Saúde sobre a COVID-19 e o Plano de Contingência para o seu enfrentamento no Risoleta. Para isso, foram produzidos boletins epidemiológicos dando visibilidade aos atendimentos, casos suspeitos, confirmados e óbitos relacionados ao coronavírus. Até o momento (desde março/2020), foram 247 publicações diárias, 17 semanais e 12 quinzenais. Também foram produzidos 58 boletins informativos sobre várias temáticas relacionadas à pandemia, incluindo orientações quanto aos Equipamentos de Proteção Individual (EPIs), experiências inovadoras de contato remoto entre pacientes isolados e familiares (visitas virtuais), homenagens às equipes pela dedicação e agradecimentos a parceiros por doações e trabalhos voluntários.

Para ampliar o acesso às notícias foi criado um grupo específico no Telegram (uso pelo celular) e adotamos um canal de divulgação inovador: a exposição de cartazes em formato de varal, no caminho para o Refeitório, em momentos pontuais e com informações estratégicas. As divulgações também circulam nos canais de comunicação internos (murais, Intranet, e-mail, WhatsApp dos líderes) e externos (site e Linkedln). Ainda, uma campanha com cards vem disseminando dicas e depoimentos de quem teve ou não a Covid-19 para conscientizar os trabalhadores e a população em geral.

E para se tornar um marco para quem venceu a doença criamos um Certificado de Vitória, que é entregue pela equipe assistencial aos usuários no momento da alta pós-Covid.

Ocorreram diversas campanhas de doação financeira, de aparelhos celulares, de EPI e outros insumos, empreendidas por entidades da sociedade civil, algumas organizadas pela UFMG e outras, resultado do movimento solidário da 
comunidade junto às equipes do Risoleta com oferta de lanches, chocolates, pizzas, buscando demonstrar gratidão pelo trabalho realizado.

\section{AS LIÇÕES APRENDIDAS COM A PANDEMIA}

A Pandemia da COVID-19 trouxe sobressaltos num cotidiano institucional marcado por processos organizacionais relativamente estáveis, apesar das frequentes superlotações no Pronto Socorro e Maternidade, orçamento restrito, absenteísmo e rotatividade de profissionais. Novos desafios se apresentaram e a premência de um processo de planejamento se impôs para permitir uma abordagem antecipada das situações de vulnerabilidade e risco para pacientes e trabalhadores.

Por se tratar de uma epidemia de grandes proporções e transmissibilidade entre as pessoas, as principais medidas de prevenção e controle exigiram mudança radical nos hábitos caseiros, comunitários e institucionais para cumprir o distanciamento social, o uso de máscaras e outros EPI e a higienização frequente das mãos. Cada trabalhador/gestor passou a responder por vigilância cotidiana em relação à sua postura, à dos colegas e junto aos pacientes assistidos.

Tornaram-se fundamentais a utilização de protocolos assistenciais e a separação dos fluxos e processos relacionados à COVID-19 para reduzir e controlar a disseminação da doença na instituição, demandando maior atenção e cuidados com rotinas e processos assistenciais, inclusive junto a públicos anteriormente não inseridos, como o pessoal da portaria, recepção e manutenção.

A estruturação da Linha de Cuidado para Pacientes Suspeitos de Covid-19 demonstrou a capacidade da comunidade hospitalar de desenvolver com agilidade e de maneira criteriosa mudanças profundas nos processos de trabalho de forma segura e sem prejuízos assistenciais para os outros perfis atendidos, demonstrando o envolvimento e compromisso das equipes, superando o medo e a insegurança gerados pelos riscos de contágio.

Ao longo desses doze meses, muitas questões foram enfrentadas e o maior aprendizado refere-se a esse grande desafio de reafirmar o compromisso do HRTN como hospital geral que necessita manter o seu papel e se adequar com agilidade e competência às novas demandas colocadas pelo contexto geral da saúde, construindo internamente uma rede de comunicação, de envolvimento e comprometimento das equipes com o novo momento.

Para isso, o grupo de lideranças institucionais produziu ações concretas de suporte técnico, organizacional e operacional capazes de conferir direcionalidade e segurança sobre o que fazer e como fazer as adequações necessárias, ofertando condições para a resolução das dificuldades e viabilização do planejado.

Nesse contexto, a comunicação constituiu-se em ferramenta fundamental de informação e esclarecimento, possibilitando canais de fala e escuta e construção de narrativas que permitiram o fortalecimento da relação de confiança entre os vários atores institucionais pela transparência e assertividade.

Como resultado houve maior aproximação do corpo dirigente do HRTN com os coordenadores, gerentes das Linhas de Cuidado assistenciais e setores de apoio, possibilitando um clima organizacional de pertencimento e solidariedade que tem animado o trabalho e as equipes por meio da capilarização das informações e dos resultados alcançados internamente, sem perder de vista a inserção na cidade e no eixo norte da região metropolitana. Trata-se de importante conquista que precisa ser valorizada pela potência de alimentar e criar novas conexões com este coletivo de trabalhadores, visando sempre animar novas possibilidades de trabalho e articulações entre as equipes.

Vale destacar ainda a solidariedade da sociedade civil e comunidade próxima neste momento de tantas atribulações. Foram muitas doações materiais e financeiras recebidas, mas 
especialmente significativo foi o carinho expresso pela população por meio de lanches e doações de alimentos nos vários turnos de trabalho que quebravam o clima, por vezes tenso, e possibilitavam compartilhamento e respiros na rotina dura do trabalho dos plantões.

É importante refletir ainda sobre os limites e fragilidades identificados no manejo dessa pandemia e que se referem, principalmente, à falta de maior articulação dos diversos equipamentos da rede, em especial, os hospitalares com a atenção primária em $\mathrm{BH}$ ou com os municípios vizinhos, não tendo sido desenvolvidas ações específicas que permitissem ampliar e potencializar as estratégias de promoção e apoio junto às comunidades. Nesse sentido, faltou capilarização do cuidado dos pacientes no pósalta com discussão para implementação da busca ativa sistemática dos egressos e seus contatos junto à APS. Faltou interlocução e troca entre os serviços para garantia de um cuidado compartilhado e articulado em rede e essa questão coloca o desafio de se criar novos mecanismos de comunicação efetiva e de transição do cuidado em rede.

Por fim, vale enfatizar que o percurso do HRTN como hospital geral com porta aberta 24 horas foi ampliado pela inclusão da Linha de Cuidado aos Pacientes de COVID-19, fortalecendo sua importância como estação cuidadora e observatório da rede do eixo norte de BH e RM. Papel esse reconhecido pelas instâncias gestoras do SUS-BH e do SUS-MG e que evidenciam o compromisso institucional de inserção plena na Rede de Atenção à Saúde, produzindo um modelo de cuidado a partir das demandas da população do território regional. 


\section{REFERÊNCIAS}

1. Franco T.B.; Magalhães Júnior, H. M. Integralidade da assistência à saúde: organização das linhas do cuidado. In: Merhy EE et al (Orgs.). O trabalho em saúde: olhando a experiência do SUS no cotidiano. São Paulo: Hucitec; 2003. p. 125-34

2. Costa MA, Mota JAC, Figueiredo RCP, Antunes MIS, Torres HOG, Coutinho AAP, Ribeiro ML. Hospital Risoleta Tolentino Neves: o desafio de produzir assistência e ensino baseados no modelo de linhas de cuidado. In: Magalhães Júnior (Org.) Desafios e Inovações na Gestão do SUS em Belo Horizonte. Belo Horizonte: Mazza Edições; 2010, p 549-570.

3. Silva S. F. Contribuição para Análise da Implantação de Redes de Atenção à Saúde no SUS. Rev. Divulgação em Saúde para Debate. 2014;(52):165-176.

4. Cecílio, L. C.O.; Merhy, E.E. O singular processo de coordenação dos hospitais. Saúde em Debate 2003; 22(64):110-22. 